\title{
AVALIAÇÃO DO EFEITO DA TEMPERATURA E DO pH NA HIDRÓLISE ENZIMÁTICA DO ÓLEO DE CRAMBE
}

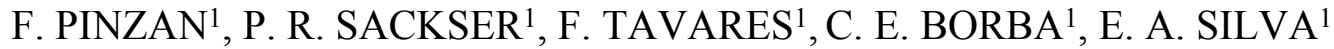 \\ ${ }^{1}$ Universidade Estadual do Oeste do Paraná, Departamento de Engenharia Química \\ E-mail para contato: fernandapinzan@hotmail.com
}

\begin{abstract}
RESUMO - A decrescente produção de petróleo incentivou os estudos na área de biocombustíveis e atualmente o biodiesel já está incorporado ao diesel. Uma alternativa para a produção do biodiesel é a hidroesterificação, que possibilita o uso de matérias-primas de alta acidez, além de produzir uma glicerina mais pura do que o processo de transesterificação. $O$ presente trabalho estudou o efeito da temperatura e do $\mathrm{pH}$ na etapa de hidrólise empregando a enzima comercial Lipozyme RM IM e óleo de crambe. As condições empregadas foram de $25 \mathrm{~g}$ de óleo de crambe, 2,2\% de enzima da massa total, razão molar água/óleo 10:1 e nível rotacional de $790 \mathrm{rpm}$. Amostras foram obtidas para um tempo reacional de 30 horas. A máxima acidez obtida utilizando água foi $64,52 \%$ à $45^{\circ} \mathrm{C}$. Porém a $28^{\circ} \mathrm{C}$ atingiu-se $64,16 \%$, concluindo-se que altas temperaturas não são necessárias para um bom rendimento da reação. Analisando o efeito do $\mathrm{pH}$, o maior índice de acidez foi $26,63 \%$ com pH 7 e os demais básicos não tiveram significativo aumento de conversão. Sendo assim, a água é suficiente para as reações de hidrólise enzimática do óleo de crambe.
\end{abstract}

\section{INTRODUÇÃO}

A maior parte da energia consumida no Brasil provém do petróleo que é uma fonte limitada e não renovável o que tem causado preocupação visto que a produção de petróleo deverá ser cinco vezes menor que a atual até 2050. Além disso, o aquecimento global incentivou uma intensa busca por combustíveis renováveis (Santos e Pinto, 2008; Puquevicz et al., 2008).

O biodiesel é uma alternativa para o diesel e é derivado de fontes renováveis como óleos vegetais e gordura animal. Ambas não podem ser utilizadas diretamente nos motores a diesel, pois a gordura animal possui grande quantidade de ácidos graxos livres que solidificam-se em temperatura ambiente, enquanto o óleo vegetal tem elevada viscosidade e baixa volatilidade além de ser poli-insaturados (Hanna et al., 2009). No entanto, tanto óleos quanto gorduras servem de matéria-prima para a produção do biodiesel que é definido como uma mistura de mono álquil ésteres de ácidos graxos (Puquevicz et al., 2008). Este combustível oferece menos desgaste do motor, melhor lubrificação e quando queimado, produz poluentes menos prejudiciais à saúde humana se comparados ao diesel (Tavares, 2014).

Dentre os vários processos utilizados para a produção de biodiesel o mais utilizado é a transesterificação homogênea a qual emprega, geralmente, hidróxido de sódio ou de potássio 
como catalisador. Com frequência, óleo vegetal ou gordura animal pode conter pequenas quantidades de água e ácidos graxos livres e, neste caso, a presença destes compostos no meio reacional pode ocasionar a formação de sabão, uma reação indesejada, pois diminui o rendimento do biodiesel e prejudica a separação dos ésteres de ácidos graxos e do glicerol. Para que tal reação não ocorra o processo exige que a matéria prima contenha percentual de acidez abaixo de 1\% (Tavares, 2014).

A hidroesterificação surgiu como processo alternativo para a produção de biodiesel, o qual permite a utilização de qualquer matéria-prima proveniente de óleo ou gordura independente do teor de água e acidez, sendo esta a principal vantagem em relação à transesterificação, uma vez que $80 \%$ dos custos de produção são relativos a matéria-prima (Encarnação, 2008). Tal processo é caracterizado por duas etapas: hidrólise e esterificação. A hidrólise consiste numa reação química entre o tri, di e monoacilglicerídeos do óleo com a água formando glicerina e ácidos graxos livres, o que ocasiona um aumento do índice de acidez da mistura final em relação a mistura inicial. Pupo et al. (2011) apresenta em detalhe o mecanismo de reação da hidrólise enzimática de óleos vegetais. A esterificação consiste numa reação química entre um ácido orgânico ou inorgânico, podendo ser os ácidos graxos livres resultantes da hidrólise, com um álcool obtendo uma mistura de monoalquil ésteres (biodiesel) e água.

O crambe (Crambe Abyssinian) se mostra interessante para a produção do biodiesel por conter elevado teor de óleo no grão, cerca de 26 a 38\% (Lunelli, 2012). Além disso, a oleaginosa tem ciclo de produção de 85 a 90 dias, caracterizando o período de entre safra das culturas principais e não requer maquinário específico. $\mathrm{O}$ crambe pertence à família das crucíferas, originária da Etiópia na região de transição entre temperada e quente (Falasca et al., 2010) sendo resistente à más condições climáticas. No Brasil, o crambe é tolerante ao déficit hídrico, ao clima rústico e pode ser plantado no período de safrinha, ou seja, no inverno (Colodetti et al., 2012).

Hidrólise pode ser realizada via catálise química ou enzimática. A química pode dar-se pelo uso de catalisadores ácidos ou básicos e a conversão é acelerada pelo aumento da temperatura, sendo que a ácida necessita de mais calor. A hidrólise pela rota enzimática o catalisador é de origem biológica e tem ação específica, sendo necessário um controle do meio reacional incluindo e a temperatura, que são mais moderadas $\left(20^{\circ} \mathrm{C}-40^{\circ} \mathrm{C}\right)$ e varia conforme a resistência do catalisador (Araújo et al., 2013). As lipases são um tipo de enzima classificadas como hidrolases que são capazes de catalisar reações de hidrólise e síntese de ésteres. A principal forma de produção de lipases é a partir de culturas de micro-organismos pelo custo reduzido e alta capacidade produtiva (Monteiro et al., 2001; Yasukawa et al., 2004). A enzima comercial Lipozyme RM IM é proveniente do micro-organismo Rhizomucor Miehei e tem se destacado nas reações de hidrólise pela boa estabilidade e alta atividade (275 IUN/g) em condições diversas (Rodrigues e Fernadez-Lafuente, 2010).

Este trabalho tem como objetivo avaliar o efeito da temperatura e do $\mathrm{pH}$ no rendimento da hidrólise enzimática do óleo de crambe.

\section{METODOLOGIA}


O óleo de crambe utilizado nas reações de hidrólise foi concedido pela Fundação MS localizada em Maracaju/MS. A enzima lipase Lipozyme RM IM foi cedida pela empresa LNF Latino Americana. Foram feitas 7 reações, onde 25 gramas de óleo de crambe serão pesadas em balança analítica diretamente em reator de vidro (Erlenmeyer - $125 \mathrm{~mL}$ ). Em seguida 2,2\%, em massa total de substrato, de enzima e água destilada (razão molar água/óleo 10:1) foram pesadas e adicionadas ao reator, essas reações foram realizadas com nível de rotação de $790 \mathrm{rpm}$, utilizando-se a chapa agitadora magnética para agitação e aquecimento. Outras 13 reações foram feitas nas mesmas condições, porém com $\mathrm{pH}$ variando de 3 a 10. Para cada experimento, passado o tempo determinado de 30 horas, as amostras foram centrifugadas a fim de separar os ácidos graxos.

As análises de acidez foram feitas por titulação com solução de $\mathrm{NaOH}$, onde cerca de 1 g de óleo e quatro gotas de solução de fenolftaleína são diluídas em $50 \mathrm{ml}$ de solução 1:1 éter/etanol. Esta solução foi titulada com uma solução de $\mathrm{NaOH}$ 0,05 M sob agitação vigorosa até mudança de coloração (mudança súbita de uma coloração branca para rosa). A acidez do óleo foi então calculada de acordo com a Equação 1.

$$
A c=100 \% \times \frac{V M_{\mathrm{NaOH}} P M_{A G L}}{m a}
$$

Onde:

$V=$ Volume (L) de solução de $\mathrm{NaOH}$ gasto na titulação;

$M_{\mathrm{NaOH}}=$ Molaridade da solução de $\mathrm{NaOH}(\mathrm{mol} / \mathrm{L})$;

$P M_{A G L}=$ Massa molar média dos ácidos graxos presentes no óleo de crambe; $m a=$ Massa da amostra de óleo $(\mathrm{g})$.

\section{RESULTADOS E DISCUSSÕES}

\subsection{Efeito da Temperatura}

Foram realizadas reações de hidrólise enzimática em diferentes temperaturas dentro da faixa de 28 a $70^{\circ} \mathrm{C}$. Na Figura 1 é apresentado o índice de acidez (\%), da mistura final obtida a partir da hidrólise enzimática do óleo de crambe.

A partir da Figura 1, verifica-se que em $45^{\circ} \mathrm{C}$ obteve-se a maior conversão, que é correspondente a $64,52 \%$. No entanto, considerando os desvios, nota-se que os três primeiros pontos são equivalentes e por isso a temperatura de $28^{\circ} \mathrm{C}$ torna-se a mais vantajosa. Em $70^{\circ} \mathrm{C}$ a baixa conversão é, provavelmente, acarretada pela desnaturação da enzima Lipozyme RM IM.

Awadallak et al., (2013) estudou a hidrólise enzimática do óleo de palma a partir da enzima comercial Lipozyme RM IM. Os experimentos requeriam $15 \mathrm{~g}$ de óleo de palma, $1,5 \mathrm{~g}$ de água e uma quantidade de enzima de $1,36 \%$ da massa total água/óleo, que eram inseridas 
no reator e conduzidas a $55^{\circ} \mathrm{C}$ por 24 horas resultando em $40 \%$ de acidez final, enquanto que no presente trabalho obteve-se, em $28^{\circ} \mathrm{C}$, uma conversão final de $64,16 \%$ num intervalo de tempo de 30 horas. Esse resultado favorece o presente estudo pela viabilidade econômica em função da redução dos gastos energéticos e maior conversão apesar das seis horas a mais de funcionamento.

Figura 1 - Efeito da temperatura na hidrólise enzimática do óleo de crambe. --- índice de acidez inicial do óleo de crambe

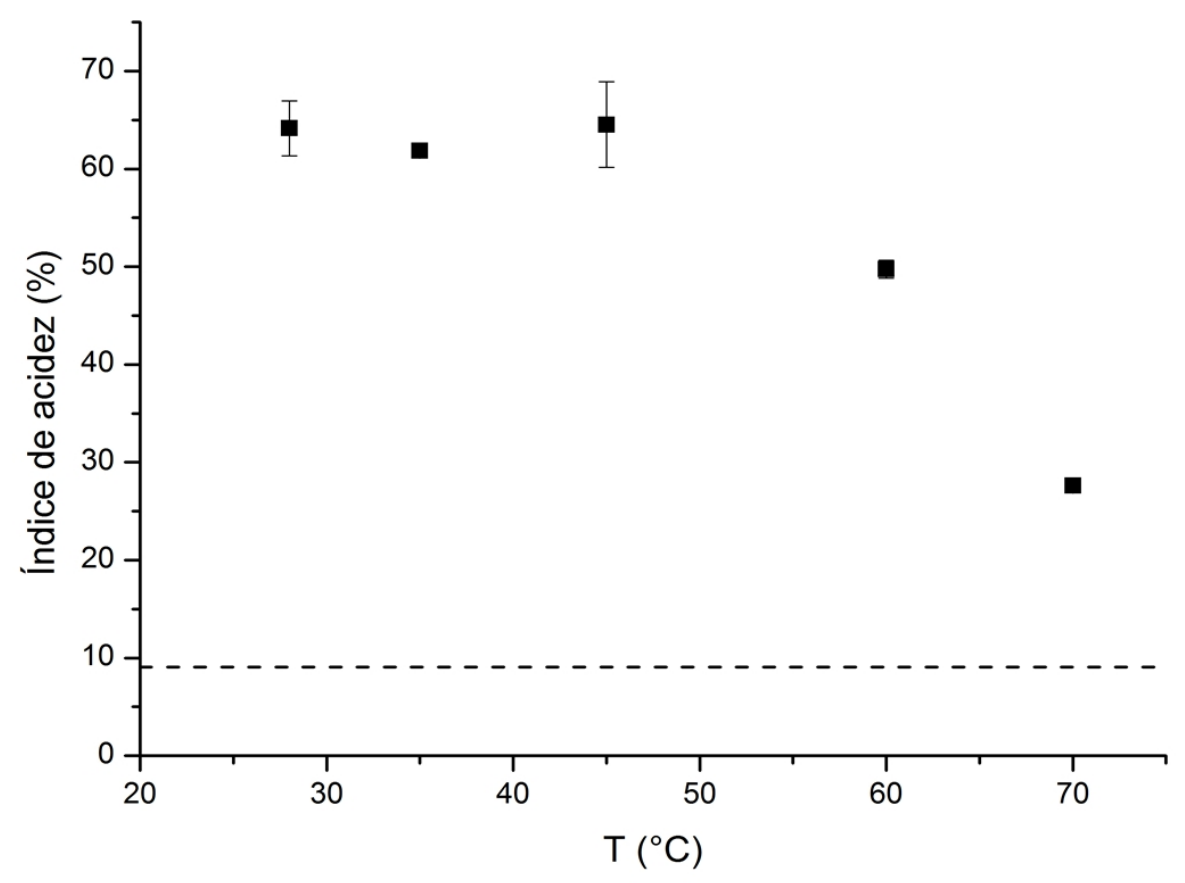

\subsection{Efeito do pH}

Para análise da influência do $\mathrm{pH}$ nas reações de hidrólise enzimática do óleo de crambe foram realizados experimentos em que o $\mathrm{pH}$ variou de 3 a 10. Na Figura 2 é apresentado o índice de acidez (\%) da mistura final.

A partir da Figura 2, nota-se um aumento do índice de acidez no pH 7 (26,63\%) e nos demais básicos, que são 8,9 e 10, a conversão permanece quase constante. Dessa forma é possível dizer que a enzima comercial Lipozyme RM IM teve melhor atividade em meio neutro ou básico. Portanto torna-se desnecessária a substituição da água por outra solução, nas reações de hidrólise enzimática do óleo de crambe.

Para Cavalheiro (2013), no estudo do efeito do pH na hidrólise do óleo de canola, os experimentos foram realizados nas condições de $5 \mathrm{mmol}$ de óleo de canola, razão molar água/óleo 3:1 e massa de enzima de 10\% da massa total de óleo. A enzima Lipozyme RM IM combinada com a Lipozyme TL IM ativam-se melhor em $\mathrm{pH}$ ácido 5,28. Mas para a segunda combinação entre RM IM e CALB, a hidrólise foi favorecida em pH básico 8,2. No entanto, neste trabalho que está sendo desenvolvido apenas com a enzima Lipozyme RM IM, o pH mais adequado foi de 7, podendo utilizar água nas reações de hidrólise. 
Figura 2 - Efeito do pH na hidrólise enzimática do óleo de crambe. --- índice de acidez inicial do óleo de crambe

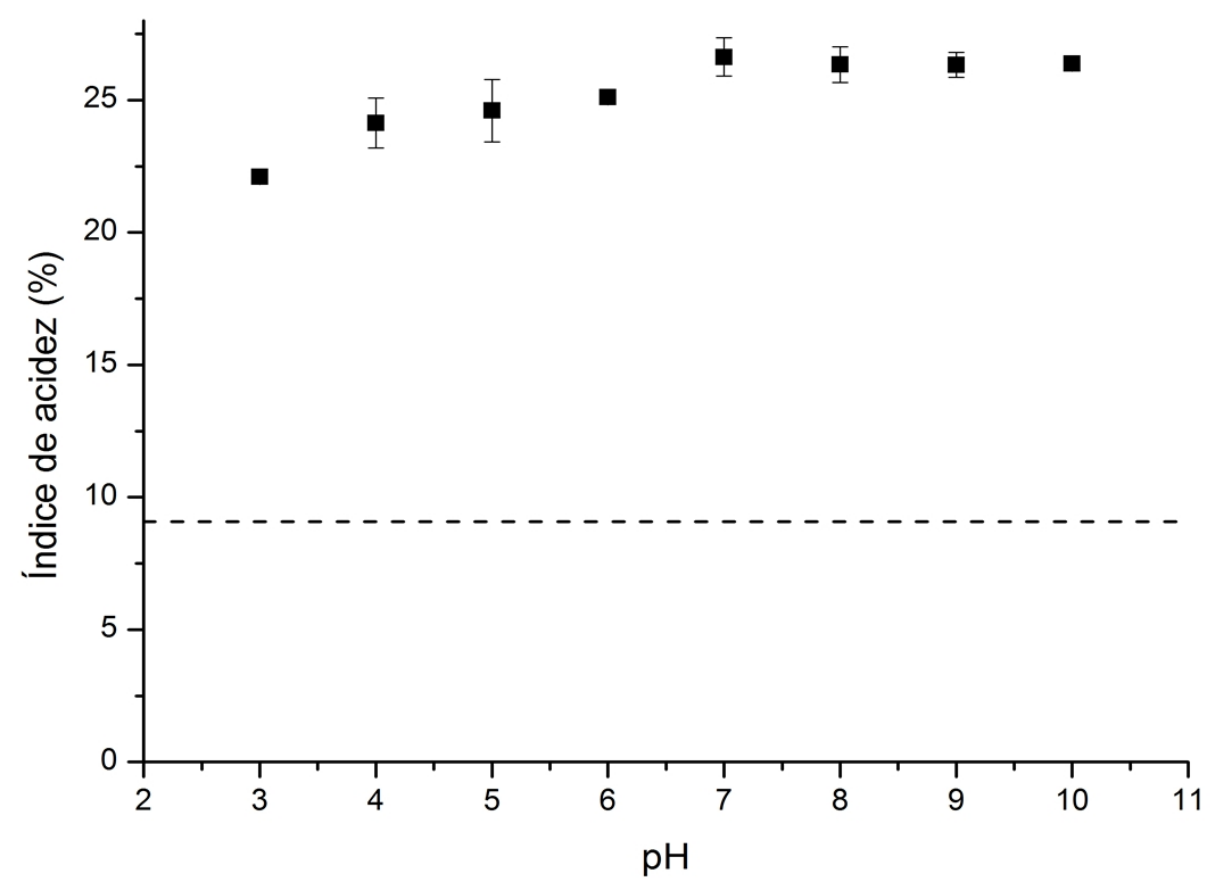

\section{CONCLUSÃO}

Com o estudo do efeito da temperatura e do $\mathrm{pH}$ na hidrólise enzimática do óleo de crambe conclui-se que a temperatura mais economicamente viável é de $28^{\circ} \mathrm{C}$, por reduzir o gasto energético. A água pode ser empregada nas reações favorecendo a atividade da enzima comercial Lipozyme RM IM, que se adapta melhor em meio neutro e básico. Estes resultados dão potencial para a produção de ácidos graxos livres a partir da hidrólise enzimática do óleo de crambe e portanto, novos estudos podem ser feitos a fim de otimizar as condições do processo.

\section{REFERENCIAS}

ARAÚJO, C. R. de.; GARRIDO, C. V. S.; SANTOS, J. M. G. M.; LEAL, S. C. S.; CAMPOS, L. M. A. Estudo das rotas de hidrólise química e biológica para a produção de etanol de segunda geração a partir de resíduos lignocelulósicos. Revista Unifacs, v. $12,2012$.

AWADALlAK, J. A.; VOLL, F.; RIBAS, M. C.; SILVA, C. da.; FILHO, L. C.; SILVA, E. A. da. Enzymatic catalyzed palm oil hydrolysis under ultrasoun irradiation: Diacylglycerol synthesis. Ultrasonics Sonochemistry, v. 20, p. 1002-1007, 2013.

COLODETTI, T. V.; MARTINS, L. D.; RODRIGUES, W. N.; BRINATE, S. B. B.; TOMAZ, M. A. Crambe: Aspectos gerias da produção agrícola. Enciclopédia Biosfera, v. 8, p. $258,2012$. 
ENCARNAÇÃO, A. N. G. Geração de biodiesel pelos processos de transesterificação e hidroesterificação, uma avaliação econômica. Dissertação de mestrado de Tecnologia de Processos Químicos da Universidade Federal do Rio de Janeiro, Rio de Janeiro, 2008.

FALASCA S. L., FLORES N., LAMAS M. C., CARBALlO S. M., ANSCHAU A. Crambeabyssinica: An almost unknown crop with a promissory future to produce biodiesel in Argentina. International Journal of Hydrogen Energy, v. 35, p. 5808-5812, 2010 .

LUNELLI, I. E. Efeitos de arranjos nutricionais de NPK na produtividade de grãos e rendimento de óleo da cultura do crambe. Dissertação de mestrado de Energia da Agricultura da Universidade Estadual do Oeste do Paraná, Cascavel, Paraná, 2012.

MONTEIRO J.B.R., ROSADO E.L. Obesidade e a substituição de macronutrientes da dieta. Revista de Nutrição, v. 14, p. 1-31, 2001.

PUPO, M. M. S. de; ALMEIDA, A. R.; MAIA, W. A.; RAMOS, A. L. D. Produção de biodiesel via hidroesterificação utilizando catalisadores ácidos. Dissertação de mestrado de Engenharia Química da Universidade Federal de Sergipe, São Cristóvão, Sergipe, 2011.

PUQUEVICZ, A. L.; DYCK, B.; MENEGOTTO, G. F. de.; PIRES, L. M. Análise comparativa da utilização de diesel e biodiesel no funcionamento de um grupo gerador. Da Vinci, v. 5, p. 179-195, 2008.

RODRIGUES, R. C., \& FERNANDEZ-LAFUENTE, R. Lipase from Rhizomucor miehei as an industrial biocatalyst in chemical process. $J$. of Molecular Catalysis B: Enzymatic, v. 64(1), p. 1-22, 2010.

SANTOS, A. P. B.; PINTO, A. C. Biodiesel: Uma alternativa de combustível limpo. Química Nova na Escola, v. 31, p. 58-62,2009.

TAVARES, F. Hidroesterificação do óleo de crambe empregando catálise enzimática. Dissertação de mestrado de Engenharia Química da Universidade Estadual do Oeste do Paraná, Toledo, Paraná, 2014.

YASUKAWA, T.; KATSURAGI, Y.; MATSUO, N.; FLICKINGER, B. D.; TOKIMITSU, I., MATLOCK, M. G. In: Diacylglycerol Oil. Champaign, Illinois: Editora: AOCS, 2004. 